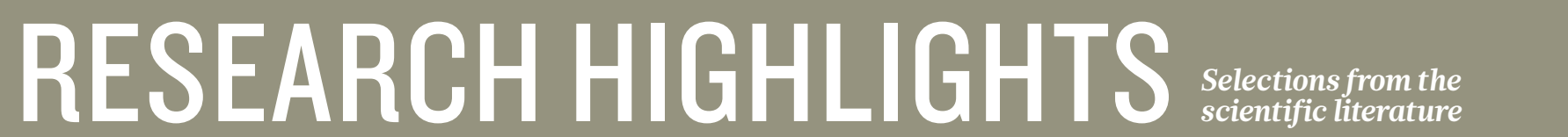

\section{MARINE METAGENOMICS}

\section{Sequencing from scratch}

Although most microorganisms cannot currently be cultured, their genomes may soon be accessible.

Until now, metagenomic analyses have been able to identify only dominant members of a microbial community or those sequenced previously. Virginia Armbrust and her group at the University of Washington in Seattle developed computational tools to tame the massive amount of data produced by next-generation sequencers. The method successfully sequenced two of 14 candidate genomes identified in samples from Puget Sound, most notably a microbe of low abundance but great interest - a representative of the mysterious, as yet uncultured organisms known as marine group II Euryarchaeota.

Researchers now have a way to peer into the secret lives of the uncultured majority.

Science 335, 587-590 (2012)

\section{MATERIALS}

\section{Printing tiny coiled antennas}

Typically, the largest circuit component in wireless electronic devices such as mobile phones is the antenna, which sends and receives electromagnetic waves. The tiniest antennas available are made up of wires twisted into three-dimensional coils to save

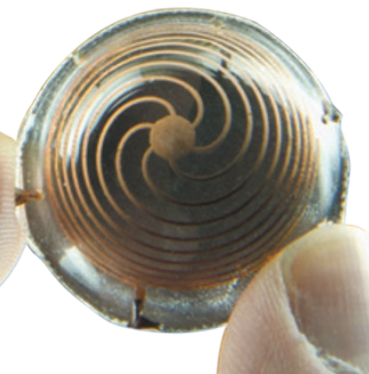

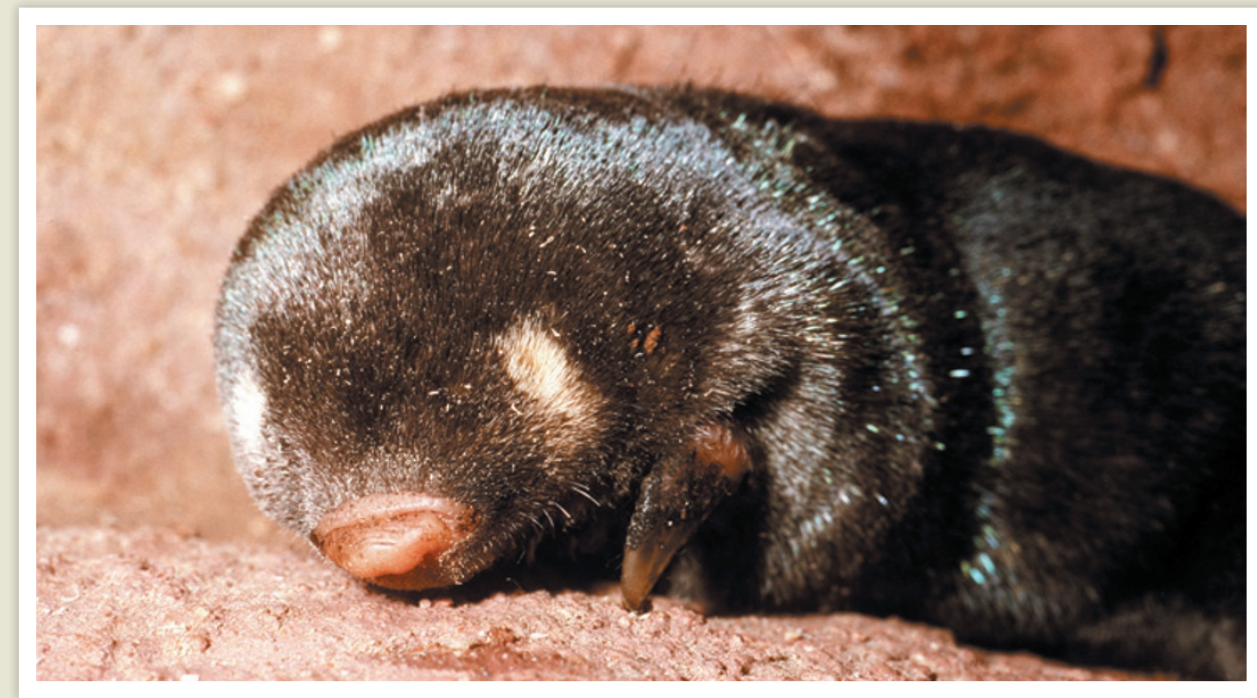

EVOLUTION

\section{Glad rags for a blind mole}

Golden moles have a blue-green sheen to their coats that is a rare example of iridescence in mammals, report Matthew Shawkey at the University of Akron in Ohio and his colleagues.

The group conducted the first detailed study of iridescent outer hairs and non-iridescent downy hairs from four species of golden mole. Iridescent hairs were highly flattened with much smaller scales than their less eye-catching counterparts. The scales form multiple layers, which alternate in colour between light and dark, and probably produce colour as light passes between layers in a phenomenon called thin-film interference.

All four mole species are blind, so it is unlikely that the hairs evolved as sexual ornamentation. The authors suggest that the iridescence of these burrowing animals is a by-product of adaptations for durable, low-friction pelts. Biol. Lett. http://dx.doi.org/10.1098/rsbl.2011.1168 (2012) on space while maintaining high radiation efficiency and wide bandwidth. But bending wires is cumbersome and expensive.

Stephen Forrest and Anthony Grbic at the University of Michigan in Ann Arbor and their colleagues report a way to rapidly transfer metallic patterns directly onto a curved polymer, which can be pre-moulded to a desired shape. Stamping the pattern onto a hemispherical polymer, for instance, produces miniature high-performance antennas curled in spherical helices (pictured).

Adv. Mater. http://dx.doi.org/10. 1002/adma/201104290 (2012)

\section{NETWORKS}

\section{Patchy}

\section{communication}

People tend to communicate with each other in bursts, exchanging clusters of messages over short time periods, and following these up with longer gaps in communication. But are these patterns simply the result of a tendency to talk more during the day and the working week?

Hang-Hyun Jo of Aalto University in Finland and his colleagues found that these temporal cycles are not sufficient to explain the bursts. They analysed 322 million mobile-phone calls between more than 5 million users over 119 days in 2007. After removing the effects of the day-night and working-week cycles, the bursts remained.

The authors suggest that the patterns reflect something fundamental in the way that people communicate. N. J. Phys. 14, 013055 (2012)

\section{GANCER DRUGS}

\section{Chemo spans generations}

Some commonly used cancer drugs not only generate mutations in treated mice, but scar the genomes of their 
offspring as well.

Radiation is known to cause genomic instability, leading to mutations that are passed down to the first- and even second-generation progeny of exposed mice. Colin Glen and Yuri Dubrova at the University of Leicester, UK, reasoned that the same could be true of DNAdamaging chemotherapies.

The duo tested three such drugs in male mice at concentrations similar to those used in humans, and found that the offspring of exposed mice harboured up to twice as many mutations as their exposed parent at the genome location studied. Moreover, mutations were present in both the copy of the genome inherited from the exposed parent and that from the unexposed parent.

Proc. Natl Acad. Sci. USA http://dx.doi.org/10.1073/ pnas.1119396109 (2012)

\section{HUMAN EVOLUTION}

\section{Hobbit small, but not stunted}

Evidence is mounting for the argument that the 'hobbit' of Flores Island was not the same species as modern humans.

The first of the 17,000-yearold Homo floresiensis fossils were discovered in 2003; since then there has been fierce debate over whether they represent a new diminutive

Homo species, or Homo sapiens with the medical condition cretinism. Peter Brown at the University of New England in Armidale, Australia, analysed $H$. floresiensis traits such as brain mass, skeletal proportions and tooth development, and compared them with those of people with cretinism.

Brown found no signs in the small-bodied, smallbrained $H$. floresiensis of the delayed growth associated with cretinism. He says that earlier studies may have confused damage caused by the fossilization process with features of the disorder.

J. Hum. Evol. http:// dx.doi.org/10.1016/j. jhevol.2011.10.011(2012)
ASTRONOMY

\section{Core-collapse and star formation}

When massive stars accumulate more iron than their centres can hold, they explode in what is known as a core-collapse supernova. Such supernovae enrich the surrounding environment with elements, seeding the formation of other stars. Astronomers have linked the number of core-collapse supernovae in a galaxy to the rate of star formation.

Maria-Teresa Botticella at the Padua Astronomical Observatory in Italy and her colleagues compared starformation estimates based on core-collapse explosions to those based on more conventional measurements of galactic brightness. They found good agreement between their method and one of the two others studied.

The authors also used their measurements to estimate the mass range over which iron-rich stars explode. The study should improve our understanding of these supernovae and may lead to a new way of studying star formation in distant galaxies. Astron. Astrophys. 537, A132 (2012)

\section{PALAEONTOLOGY}

\section{Early bird was black}

The plumage of the world's first known bird contained at least some black, researchers report.

A team led by Ryan Carney at Brown University in Providence, Rhode Island, examined a fossilized feather (pictured) from the bird Archaeopteryx, which lived 150 million years ago. Using electron microscopy, they spotted rod-shaped, pigmented organelles called melanosomes inside preserved cells. Statistical comparison of the shape of these organelles with those of 87 extant birds identified similarities to melanosomes from birds with black plumage.

COMMUNITY CHOICE

The most viewed papers in science

\section{NUCLEAR ENERGY}

\section{Testing the waters for radionuclides}

\section{HIGHLY READ \\ on pubs.acs.org in December}

A relatively reassuring study about radioactive particles released into the ocean as a result of the accident at Japan's Fukushima Daiichi nuclear power plant

last March has proved popular reading.

Ken Buesseler at the Woods Hole Oceanographic Institution in Massachusetts and his colleagues gathered data on caesium and iodine isotopes collected after the accident by the Tokyo Electric Power Company and the Ministry of Education, Culture, Sports, Science and Technology, and compared these with pre-accident measurements for the same isotopes. Radionuclide levels peaked one month after the accident, owing partly to releases of cooling sea water used to manage the accident.

Ultimately, the team predicts "minimal impact on marine biota or humans", but suggests that more study is warranted, especially on potential radionuclide accumulation in seafood. Environ. Sci. Technol. 45, 9931-9935 (2011)

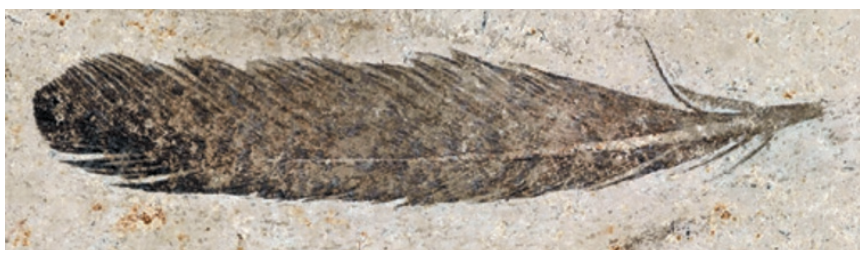

The melanin responsible for black pigmentation provides structural support as well as colour. The authors suggest that this would have improved the feathers' strength and durability - an advantage during this early evolutionary stage of dinosaur flight.

Nature Commun. http://dx.doi. org/10.1038/ncomms1642 (2012)

\section{NANOTECHNOLOGY}

\section{Electrons explain zeolite complexity}

A potentially useful catalyst with a porous structure akin to that of nanoscale Swiss cheese has had its structure revealed by electron crystallography.

Zeolites are microporous aluminosilicates with many applications, but their small size and the intergrowth of their crystals can make it difficult to determine the details of their structures.

Xiaodong Zou of Stockholm University, Avelino Corma at the Polytechnic University of Valencia in Spain and their team collected high-resolution transmission electron microscopy images and data on electron diffraction for a kind of zeolite called ITQ-39. From this, they determined the three-dimensional structure of the material - the most complex zeolite structure ever elucidated - and found that it is made up of three different arrangements of the same basic layer. They say that its unusual intersecting channel system makes it a promising catalyst for converting naphtha to diesel. Nature Chem. http://dx.doi. org/10.1038/nchem.1253 (2012)

\section{NATURE.COM}

For the latest research published by Naturevisit:

www.nature.com/latestresearch 